\title{
The influence of non-breathing-related sleep fragmentation on cognitive function in patients with cerebral small vessel disease
}

This article was published in the following Dove Medical Press journal: Neuropsychiatric Disease and Treatment

\author{
Jihui Wangl,* \\ Xiaodong Chen ${ }^{2, *}$ \\ Jinchi Liao ${ }^{2}$ \\ Li Zhou ${ }^{3}$ \\ Siyuan Liao ${ }^{2}$ \\ Yilong Shan ${ }^{2}$ \\ Zhengqi Lu \\ Jiong Tao' \\ 'Department of Psychiatry, \\ The Third Affiliated Hospital, Sun \\ Yat-sen University, Guangzhou, \\ 510630, People's Republic of \\ China; ${ }^{2}$ Department of Neurology, \\ The Third Affiliated Hospital, Sun \\ Yat-sen University, Guangzhou, \\ 5 I0630, People's Republic of China; \\ ${ }^{3}$ Department of Rehabilitative \\ Medicine, The First Affiliated Hospital \\ of Clinical Medicine of Guangdong \\ Pharmaceutical University, Guangzhou, \\ 510080 , People's Republic of China \\ *These authors contributed equally \\ to this work
}

Correspondence: Zhengqi Lu Department of Neurology, The Third Affiliated Hospital, Sun Yat-sen University, No.60, Tianhe Road, Tianhe District, Guangzhou 510630, People's Republic of China

Tel +86208525 3032

Email lzqI828@outlook.com

Jiong Tao

Department of Psychiatry, The Third

Affiliated Hospital, Sun Yat-sen

University, No.60, Tianhe Road, Tianhe

District, Guangzhou 510630, People's

Republic of China

Tel +862085253129

Email ti2023@।63.com
Background: Cognitive impairment in patients with cerebral small vessel disease (CSVD) is common, but the pathogenic mechanism is not well understood. The situation of non-breathingrelated sleep fragmentation in CSVD patients and its influence on cognitive impairment is not clear. The aim of this study was to investigate the influence of non-breathing-related sleep fragmentation on cognitive function in patients with CSVD.

Methods: A group of 89 CSVD patients without breathing-related sleep disorders in the Department of Neurology, Third Affiliated Hospital of Sun Yat-sen University was enrolled. The patients underwent magnetic resonance scan, polysomnography, cognitive function evaluation using Montreal Cognitive Assessment scale (MoCA), and Mini-Mental State Examination. The patients were assigned to study group (arousal index $[\mathrm{ArI}] \geq 26.8$ /hour) or control group (ArI $<26.8$ /hour) based on the average level of ArI (mean $=26.8, \mathrm{SD}=7.5$ ) at night, and the cognitive function of the patients in the two groups was analyzed.

Results: The total MoCA score, the subscale scores of visuospatial ability and delayed recall in the study group were significantly lower than that in the control group $(P<0.05)$. The cognitive impairment measured by MoCA was positively related to ArI level and $\% \mathrm{~N}-3$ sleep according to the results of logistic regression $(P<0.05)$.

Conclusion: Non-breathing-related sleep fragmentation is associated with cognitive impairment in CSVD patients, especially executive function and delayed recall ability.

Keywords: cerebrovascular disorders, sleep, fragmentation, cognition, polysomnography

\section{Introduction}

Continuous, undisturbed sleep at night is significantly beneficial for one's memory, immune regulation, and removal of neurotoxic byproducts that accumulate in the central nervous system during the awake period. ${ }^{1}$ Sleep fragmentation, characterized by recurrent awakenings after sleep onset, often affects the elderly ${ }^{2}$ and could cause functional or even organic damage of the brain. ${ }^{3}$ Sleep fragmentation can be breathingrelated or non-breathing-related. Non-breathing-related sleep fragmentation is sleep fragmentation caused by reasons other than breathing problems, for example age, cerebrovascular diseases, Alzheimer's, dementia, Parkinson's disease, etc. ${ }^{4}$

Cerebral small vessel disease (CSVD) is a syndrome of clinical and imaging findings that are thought to result from pathologies in perforating cerebral arterioles, capillaries, and venules, it is the most common cause of vascular cognitive impairment and dementia. ${ }^{5}$ The pathogenic mechanism of cognitive impairment is not yet fully understood at the moment. ${ }^{6}$ Research shows that non-breathing-related sleep fragmentation is related to pathological changes in iconology in CSVD, ${ }^{7}$ 
but there is still no report on the relationship between non-breathing-related sleep fragmentation and cognitive impairment in this kind of patient. This research studied patients' cognitive impairment affected by the severity of non-breathing-related sleep fragmentation, to help further investigate the pathological mechanism of CSVD-related cognitive impairment and to provide more evidence of CSVD prevention and treatments.

\section{Methods}

\section{Design}

This was a cross-sectional study. Patients were enrolled in The Third Affiliated Hospital of Sun Yat-sen University from August 2017 to July 2018. The study was approved by the ethics committee of the hospital, and was conducted according to the Declaration of Helsinki. All participants provided written informed consent.

\section{Participants}

One hundred and four consecutive patients with symptomatic CSVD were screened for recruitment following the inclusion/ exclusion criteria detailed in the next paragraph.

Inclusion criteria were: 1) patients aged 35-70 years; 2) with one or more magnetic resonance (MR) markers of CSVD: lacunar infarcts (LI), white matter hyperintensities (WMH), cerebral microbleeds (CMB), and visible perivascular spaces (PVS); ${ }^{8} 3$ ) without larger subcortical or cerebral Water-shed infarctions $(>1.5 \mathrm{~cm})$ on magnetic resonance imaging (MRI) as these are often embolic; 4) without large artery disease (carotid, vertebral, or intracranial stenosis $>50 \%$ ); 5) apnea-hypopnea index (AHI) $<5 /$ hour based on polysomnography (PSG).

Exclusion criteria were: 1) severe mental disorders such as schizophrenia, bipolar disorder or major depression, severe somatic or neurological disorders; dementia due to congenital mental disorders and other diseases; 2) presence of DSM-V Breathing-Related Sleep Disorders; 3 ) taking drugs that affect cognition or sleep; 4) unable to complete the scales due to objective conditions, such as deafness, hemiplegia, aphasia, visual impairment, etc; and 5) being pregnant.

\section{Brain MRI scan}

Patients underwent brain MRI on a 3-Tesla scanner (GE Discovery MR750) with a standard 8-channel head coil. Sequences included 3D TOF-MRA, T1Flair, T2WI, T2Flair, and SWI. Brain MRI was rated for the presence of four SVD makers: LI, WMH, CMB, and PVS independently.
The presence of each SVD feature was summed in an ordinal "SVD burden score" (range 0-4). ${ }^{8}$

\section{PSG evaluation}

All patients were evaluated for two consecutive nights in a sleep laboratory. The wireless telemetry PSG system (SOMNOscreen plus PSG+; SOMNOmedics GmbH, Randersacker, Germany, analysis software "DOMINO") was used to monitor sleep. Sleep recordings were scored by an experienced PSG technologist blinded to the group assignments. The data were analyzed according to the American Academy of Sleep Medicine Manual for the Scoring of Sleep and Associated Events V.2.0. ${ }^{9}$ PSG measures reported include: sleep onset latency (length of time in minutes that it takes to sleep); total sleep time (total duration of sleep in hours during the PSG recording); sleep efficiency ([SE] total sleep time divided by time in bed); wake after sleep onset ([WASO] the number of minutes the participant was awake after onset of persistent sleep); percentage of each sleep stage (N-1, N-2, N-3 [slow wave sleep] and rapid eye movement [REM] sleep); apnea-hypopnea index ([AHI] \% time oxygen saturation $<90 \%$ ); and arousal index ([ArI] total number of arousals divided by the duration of sleep in hours. An arousal was defined as any sudden increase in electroencephalography frequency [to alpha or theta] that lasted $>3$ seconds). ${ }^{10}$

\section{Cognitive function measurement} Montreal Cognitive Assessment scale (MoCA)

The Chinese version of MoCA was used to evaluate cognitive function regarding aspects of visuospatial ability, executive function, naming, attention, language, abstraction, delayed recall, and orientation, with a total score between 0 and 30 . If the educational level is $<12$ years, one point is added. ${ }^{11,12}$ Referring to the results of domestic research in People's Republic of China, the cutoff point to identify cognitive impairment in CSVD is $<23$ points. ${ }^{13}$

\section{Mini-Mental State Examination (MMSE)}

The Chinese version of MMSE is a 30-point questionnaire that is used to measure cognitive impairment regarding aspects of registration (repeating named prompts), attention and calculation, recall, language, ability to follow simple commands, and orientation. Any score $\geq 24$ points (out of 30) indicates a normal cognition. Below this, scores can indicate severe ( $\leq 9$ points), moderate (10-18 points), or mild (19-23 points) cognitive impairment. ${ }^{14}$ 


\section{Statistical methods}

Statistical analyses for the present study were carried out with the SPSS software package (version 20.0). Descriptive statistics were presented as mean \pm SD for continuous variables and as numbers or proportions for categorical variables. Student's $t$-tests or Mann-Whitney was used when appropriate to compare the differences in sleep parameters and cognitive functions between the two groups. Binary logistic regression analyses were performed to assess the associations between total MoCA scores and sleep parameters (PSG). Regarding dependent variables, the total MoCA scores were dichotomized and coded as "dependent-1" $(<23)$ and "independent-0" ( $\geq 23)$. The independent variables included SE, REM sleep ratio, N-3 sleep ratio, and WASO, which were included as continuous variables and ArI, coded as "dependent- 0 " ( $<$ mean) and "independent- 1 " ( $\geq$ mean) as categorical variables in the models. Two-sided $P$-values were considered to be statistically significant at $\leq 0.05$.

\section{Results}

\section{Clinical characteristics of patients}

One hundred and four consecutive patients with symptomatic CSVD were screened for recruitment. Fifteen patients were excluded because they: 1) had breathing-related sleep disorders $(n=10) ; 2)$ had delayed sleep phase disorder $(n=1)$; and 3 ) had severe comorbid medical conditions $(n=4)$. A group of 89 patients was thus recruited of which 56 patients met chronic insomnia criteria. The flow diagram of the study is presented in Figure 1. The mean of ArI for all the patients was 26.8/hour (mean ArI $=26.8, \mathrm{SD}=7.5$ ). Patients were divided into study group (ArI $\geq 26.8$ /hour) and control group (ArI $<26.8$ /hour).

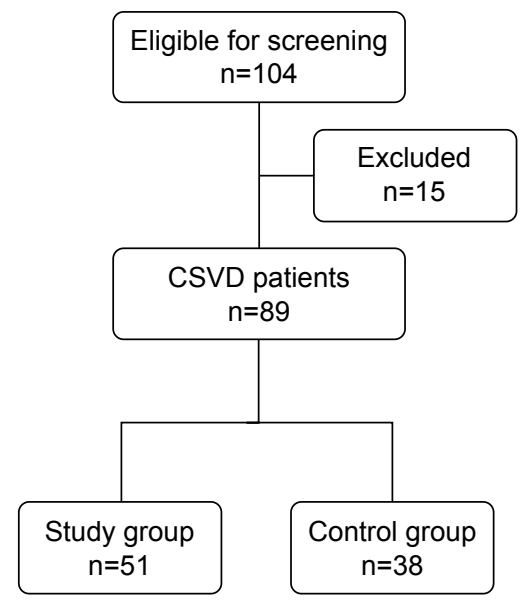

Figure I Flow diagram of the study. Abbreviation: CSVD, cerebral small vessel disease.
There were no significant differences in demographic data, comorbidities, and SVD burden score between the two groups $(P>0.05)$, as shown in Table 1.

\section{Comparison of PSG parameters between the two groups}

SE, REM ratio, and N-3 ratio of the patients in study group were lower than those in control group; WASO, N-1, and $\mathrm{N}-2$ ratio of the patients in the study group were higher than those in the control group $(P<0.05)$, with large effect sizes, as shown in Table 2.

\section{Comparison of MMSE scores between the two groups}

There was no significant difference in total score and subscale scores between study group and control group $(P>0.05)$, as shown in Table 3.

\section{Comparison of MoCA scores between the two groups}

The total score of MoCA, subscale scores of visuospatial ability and delayed recall in the study group were lower than those in the control group, with moderate to large effect sizes, as shown in Table 4.

\section{The associations between MoCA total scores and sleep parameters (PSG) based on binary logistic regression analyses}

According to the results of logistic regression, those patients with higher ArI level were 3.542 (95\% CI: 1.848-5.236, $P<0.001)$ times more likely to have cognitive impairment than those with relatively lower ArL level. Those patients with shorter duration of N-3 sleep were 1.713 (95\% CI: 1.251 $2.517, P<0.05$ ) times more likely to have cognitive impairment than those with relatively longer duration of N-3 sleep.

\section{Discussion}

Obstructive sleep apnea-hypopnea syndrome (OSAHS) is a common cause for sleep fragmentation and cognitive disorders. ${ }^{15}$ This study showed, at first screening, only ten met OSAHS diagnostic criteria out of 104 CSVD patients, but $54 \%$ met chronic insomnia criteria. This ratio is much higher than that in older adults, ${ }^{16}$ which means non-breathing-related sleep symptoms are more common in CSVD patients. The anatomy of the sleep-wake regulation system involves multiple areas of the brain, for example reticular ascending 
Table I Demographic and clinical data

\begin{tabular}{|l|l|l|l|l|}
\hline Variables & Study group $(\mathbf{n}=\mathbf{5} \mathbf{I})$ & Control group $(\mathbf{n}=\mathbf{3 8})$ & $\mathbf{t} / \mathbf{Z} / \chi^{2}$ & $P$-value \\
\hline Age (years) & $62.43 \pm I I .64$ & $61.73 \pm I 0.52$ & $\mathrm{t}=0.644$ & 0.687 \\
\hline Body mass index & $23.65 \pm 4.53$ & $22.63 \pm 3.43$ & $\mathrm{t}=0.642$ & 0.563 \\
\hline Male & $22(43.1)$ & $15(39.5)$ & $\chi^{2}=0.008$ & 0.909 \\
\hline Married & $45(88.2)$ & $34(12.9)$ & $\chi^{2}=0.033$ & 0.855 \\
\hline Educational $\leq 12$ years & $31(60.8)$ & $25(65.8)$ & $\chi^{2}=0.234$ & 0.629 \\
\hline Hypertension & 26 & 24 & $\chi^{2}=1.312$ & 0.252 \\
\hline Diabetes & 21 & 14 & $\chi^{2}=0.112$ & 0.738 \\
\hline Hypercholesterolemia & 29 & 19 & $\chi^{2}=0.171$ & 0.679 \\
\hline Smoking & $\mathrm{II}$ & 7 & $\chi^{2}=0.134$ & 0.715 \\
\hline Insomnia & 35 & 21 & $\chi^{2}=1.667$ & 0.197 \\
\hline CSVD burden score & $\mathrm{I} .52 \pm \mathrm{I} .0 \mathrm{I}$ & $\mathrm{I} .45 \pm 1.25$ & $\mathrm{Z}=0.584$ & $0.62 \mathrm{I}$ \\
\hline
\end{tabular}

Note: Data are presented as mean \pm SD, or $n(\%)$.

Abbreviation: CSVD, cerebral small vessel disease.

Table 2 Comparison of PSG parameters between the two groups

\begin{tabular}{|l|l|l|l|l|l|l|}
\hline Groups & $\mathbf{n}$ & SOL & TST & SE & WASO & LREM \\
\hline Control group & 38 & $19.23 \pm I 5.53$ & $6.48 \pm I .93$ & $0.84 \pm 0.10$ & $48.35 \pm 35.54$ & $93.63 \pm 46.53$ \\
Study group & $5 \mathrm{I}$ & $22.56 \pm 20.33$ & $6.12 \pm 2.53$ & $0.73 \pm 0.13$ & $117.34 \pm 67.55$ & $142.53 \pm 95.49$ \\
t/Z & & 0.754 & 0.396 & 2.671 & 2.957 & 1.574 \\
$P$ & & 0.627 & 0.695 & 0.012 & 0.021 & 0.099 \\
Cohen's d & & 0.18 & 0.16 & 0.93 & 1.23 & 0.62 \\
\hline Groups & $\mathbf{n}$ & REM (\%) & NREM-I (\%) & NREM-2 (\%) & NREM-3 (\%) & AHI \\
\hline Control group & 38 & $17.43 \pm 4.53$ & $8.11 \pm 3.56$ & $49.47 \pm 12.64$ & $21.53 \pm 12.64$ & $3.14 \pm 2.24$ \\
Study group & $5 \mathrm{I}$ & $12.75 \pm 5.77$ & $14.14 \pm 5.37$ & $58.96 \pm I 1.73$ & $11.73 \pm 7.42$ & $3.74 \pm 1.99$ \\
t/Z & & 2.137 & 2.954 & 2.467 & 2.054 & 0.317 \\
$P$ & & $0.04 I$ & 0.006 & 0.037 & 0.042 & 0.425 \\
Cohen's d & & 0.88 & 1.29 & 0.87 & 0.98 & 0.29 \\
\hline
\end{tabular}

Note: Data are presented as mean \pm SD, or $n(\%)$.

Abbreviations: PSG, polysomnography; SOL, sleep onset latency; TST, total sleep time; SE, sleep efficiency; WASO, wake after sleep onset; REM, rapid eye movement; NREM, non-rapid eye movement; AHI, apnea-hypopnea index; LREM, latency of rapid eye movement.

Table 3 Comparison of Mini-Mental State Examination scores between the two groups

\begin{tabular}{|l|l|l|l|l|l|l|l|l|}
\hline Groups & $\mathbf{n}$ & Total score & Orientation & Memory & Calculation & Recall & Language & Visuospatial ability \\
\hline Control group & 38 & $27.14 \pm 3.45$ & $9.64 \pm 0.95$ & $2.93 \pm 0.25$ & $4.13 \pm 1.35$ & $2.17 \pm 1.28$ & $7.47 \pm 0.64$ & $0.80 \pm 0.41$ \\
Study group & $5 \mathrm{I}$ & $26.24 \pm 2.93$ & $9.26 \pm 0.78$ & $2.91 \pm 0.23$ & $3.94 \pm 1.21$ & $1.98 \pm 0.92$ & $7.37 \pm 0.76$ & $0.78 \pm 0.42$ \\
Z & & 0.364 & 0.352 & 0.129 & 0.423 & 1.128 & 0.803 & $0.15 \mathrm{I}$ \\
P & & 0.728 & 0.727 & 0.898 & 0.676 & 0.268 & 0.428 & $0.88 \mathrm{I}$ \\
Cohen's d & & 0.28 & 0.44 & 0.08 & 0.15 & 0.17 & 0.14 & 0.05 \\
\hline
\end{tabular}

Notes: Data are presented as mean \pm SD, or $n(\%)$.

Table 4 Comparison of Montreal Cognitive Assessment scale scores between the two groups

\begin{tabular}{|l|l|l|l|l|l|l|l|l|l|}
\hline Groups & $\mathbf{n}$ & Total score & Visuospatial ability & Naming & Attention & Language & Abstract & Recall & Orientation \\
\hline Control group & 38 & $21.20 \pm 3.22$ & $2.64 \pm I .17$ & $2.33 \pm 0.85$ & $5.15 \pm 1.36$ & $1.79 \pm 1.43$ & $0.82 \pm 0.76$ & $2.93 \pm 1.21$ & $5.54 \pm 1.55$ \\
Study group & $5 \mathrm{I}$ & $17.24 \pm 4.78$ & $1.81 \pm I .55$ & $2.13 \pm 0.74$ & $5.08 \pm 1.75$ & $1.74 \pm 0.92$ & $0.69 \pm 0.74$ & $1.32 \pm 0.99$ & $4.47 \pm 0.98$ \\
Z & & 2.468 & 2.943 & 1.078 & 0.064 & 0.567 & 0.853 & 3.575 & 0.732 \\
$P$ & & 0.030 & 0.016 & 0.532 & 0.864 & 0.645 & 0.435 & 0.021 & 0.474 \\
Cohen's d & & 0.94 & 0.59 & 0.21 & 0.04 & 0.04 & 0.17 & 1.47 & 0.85 \\
\hline
\end{tabular}

Notes: Data are presented as mean \pm SD, or $\mathrm{n}(\%)$. 
activation system, nucleus raphe, locus coeruleus, hypothalamus, thalamic reticular nucleus, basal forebrain, cerebral cortex, etc. ${ }^{17}$ Small, deep cerebral vessels of CSVD patients show sclerosis, twisting, and self-regulating damage under the influence of vascular risk factors, which cause diffuse subcortical ischemia and periventricular fibrosis in the deep part of the brain and affects the sleep-related neural circuit, causing sleep disorder symptoms. ${ }^{18}$ Although this study did not have a healthy control group, a past study showed an average ArI of older adults of 21.9/hour, ${ }^{19}$ the average ArI of our CSVD subjects was 26.8 /hour, showing a significant difference, which means non-breathing-related sleep fragmentation in CSVD is severe.

The relationship between non-breathing-related sleep disorders and cognitive function is controversial; there have been both positive ${ }^{20}$ and negative results. ${ }^{21}$ The reason for this, apart from study design, might be the heterogeneity of sleep disorders. If we look further into the specific sleep parameters, some consistencies will emerge. For example, sleep duration (short or long sleep duration), WASO, and SE were found to be positively related to cognitive impairment. ${ }^{22}$ Prolonged WASO and low SE could be the result of sleep fragmentation. This study discovered that sleep fragmentation was not only related to cognitive damage, but also directly related to WASO, SE, and changes in sleep structure, which indicates that sleep fragmentation could be a relatively stable sleep parameter for predicting cognitive impairment in CSVD patients.

In this study, the difference in cognitive functioning between the two groups was mainly reflected in two aspects: delayed recall and visuospatial ability. The impairment of both cognitive abilities could be related to sleep fragmentation-induced sleep structure disturbance. Non-rapid eye movement, especially N-3 sleep is crucial to declarative memory, while REM is important for enhancing short-term memory. ${ }^{23,24}$ The test of delayed recall in MoCA, which is one kind of declarative memory, is also relevant to shortterm memory enhancing. The study group showed reduced N-3 sleep and REM ratio and impaired delayed recall, which indicates sleep fragmentation causing declarative memory impairment through sleep structure disturbance. Past experiments reported that sleep continuity disruption directly damaged the abilities of inhibitory control and working memory of healthy, senile people. ${ }^{25}$ Inhibitory control is the ability to inhibit reacting to irrelevant stimulation when pursuing a specific cognitive goal. Working memory is temporary storage of information and correlates with many more general abilities and is linked to basic sensory processes. ${ }^{26}$ This study found that the visuospatial ability impairment of the study group was significantly more serious than that of the control group, which may be the direct consequence of sleep fragmentation, because visuospatial ability is one of the main components of executive function.

\section{Conclusion}

This study indicates that non-breathing-related sleep fragmentation is common in patients with CSVD and could impair the ability of delayed recall and executive function. We suggest that reducing sleep fragmentation should be considered an important target in the prevention and treatment of cognitive impairment in CSVD patients. There are several limitations to the present study. First, because of the relatively small sample size, we did not conduct a multivariate analysis of sleep parameters and various cognitive fields, which may have led to confounding factors. We will increase the sample size for further analysis in future studies. Second, periodic leg movement is considered to be related to arousal during sleep, but it was not evaluated in this study because there were no participants who reported symptoms of restless leg syndrome. Finally, to improve our future studies, we will: 1) set up a normal control group and 2) prospectively observe changes in sleep fragmentation through treatment in CSVD patients and their effect on cognitive performance.

\section{Disclosure}

The authors report no conflicts of interest in this work.

\section{References}

1. Cedernaes J, Osorio RS, Varga AW, Kam K, Schiöth HB, Benedict C. Candidate mechanisms underlying the association between sleep-wake disruptions and Alzheimer's disease. Sleep Med Rev. 2017;31:102-111. doi:10.1016/j.smrv.2016.02.002

2. Huang YL, Liu RY, Wang QS, Van Someren EJ, Xu H, Zhou JN. Ageassociated difference in circadian sleep-wake and rest-activity rhythms. Physiol Behav. 2002;76(4-5):597-603.

3. Tononi G, Cirelli C. Sleep and the price of plasticity: from synaptic and cellular homeostasis to memory consolidation and integration. Neuron. 2014;81(1):12-34. doi:10.1016/j.neuron.2013.12.025

4. Stern AL, Naidoo N. Wake-active neurons across aging and neurodegeneration: a potential role for sleep disturbances in promoting disease. Springer Plus. 2015;4(25). doi:10.1186/s40064-014-0777-6

5. Pantoni L. Cerebral small vessel disease: from pathogenesis and clinical characteristics to therapeutic challenges. Lancet Neurol. 2010;9(7): 689-701. doi:10.1016/S1474-4422(10)70104-6

6. Shi Y, Wardlaw JM. Update on cerebral small vessel disease: a dynamic whole-brain disease. Stroke Vasc Neurol. 2016;1(3):83-92. doi:10.1136/ svn-2016-000035

7. Del Brutto OH, Mera RM, Zambrano M, Lama J, Del Brutto VJ, Castillo PR. Poor sleep quality and silent markers of cerebral small vessel disease: a population-based study in community-dwelling older adults (The Atahualpa project). Sleep Med. 2015;16(3):428-431. doi:10.1016/j. sleep.2014.10.023

8. Staals J, Makin SD, Doubal FN, Dennis MS, Wardlaw JM. Stroke subtype, vascular risk factors, and total MRI brain small-vessel disease burden. Neurology. 2014;83(14):1228-1234. doi:10.1212/ WNL.0000000000000837 
9. Rosenberg RS, Van Hout S. The American Academy of Sleep Medicine inter-scorer reliability program: sleep stage scoring. J Clin Sleep Med. 2013;9(1):81-97.

10. American Sleep Disorders Association. EEG arousals: scoring rules and examples: a preliminary report from the Sleep Disorders Atlas Task Force of the American Sleep Disorders Association. Sleep. 1992;15(2):173-184.

11. Nasreddine ZS, Phillips NA, Bédirian V, et al. The montreal cognitive assessment, MoCA: a brief screening tool for mild cognitive impairment. J Am Geriatr Soc. 2005;53(4):695-699. doi:10.1111/j.1532-5415. 2005.53221.x

12. Wen HB, Zhang ZX, Niu FS, Li L. The application of Montreal cognitive assessment in urban Chinese residents of Beijing. Zhonghua Nei Ke Za Zhi. 2008;47(1):36-39.

13. Kong FB, Yang F, Chen W, Zhao RL. Value of the Montreal cognitive assessment for the detection of vascular cognitive impairment in cerebral small vessel disease. Chin J Clinicians. 2011;5(23):6975-6980. Chinese.

14. Wang ZY, Zhang MY, Zhai GY, Chen JX, Zhao J. Application of Chinese version of Concise Mental State Examination (MMSE). Shanghai Arch Psychiatry. 1989;7(3):108-111. Available from: http://www. sinomed.ac.cn/zh/detail.do?ui=1994166490. Accessed April 8, 2019.

15. Polsek D, Gildeh N, Cash D, et al. Obstructive sleep apnoea and Alzheimer's disease: in search of shared pathomechanisms. Neurosci Biobehav Rev. 2018;86:142-149. doi:10.1016/j.neubiorev.2017.12.004

16. Patel D, Steinberg J, Patel P. Insomnia in the elderly: a review. J Clin Sleep Med. 2018;14(6):1017-1024. doi:10.5664/jcsm.7172

17. Schwartz MD, Kilduff TS. The neurobiology of sleep and wakefulness. Psychiatr Clin North Am. 2015;38(4):615-644. doi:10.1016/j. psc.2015.07.002
18. Cheng CY, Tsai CF, Wang SJ, Hsu CY, Fuh JL. Sleep disturbance correlates with white matter hyperintensity in patients with subcortical ischemic vascular dementia. J Geriatr Psychiatry Neurol. 2013;26(3): 158-164. doi:10.1177/0891988713493503

19. Bonnet MH, Arand DL. EEG arousal norms by age. J Clin Sleep Med. 2007;3(3):271-274.

20. Chen PL, Lee WJ, Sun WZ, Oyang YJ, Fuh JL. Risk of dementia in patients with insomnia and long-term use of hypnotics: a populationbased retrospective cohort study. PLoS One. 2012;7(11):e49113. doi:10.1371/journal.pone.0049113

21. Foley D, Monjan A, Masaki K, et al. Daytime sleepiness is associated with 3-year incident dementia and cognitive decline in older JapaneseAmerican men. J Am Geriatr Soc. 2001;49(12):1628-1632.

22. Yaffe K, Falvey CM, Hoang T. Connections between sleep and cognition in older adults. Lancet Neurol. 2014;13(10):1017-1028. doi:10.1016/ S1474-4422(14)70172-3

23. Ackermann S, Rasch B. Differential effects of non-REM and REM sleep on memory consolidation? Curr Neurol Neurosci Rep. 2014;14(2):430. doi:10.1007/s11910-013-0430-8

24. Abel T, Havekes R, Saletin JM, Walker MP. Sleep, plasticity and memory from molecules to whole-brain networks. Curr Biol. 2013;23(17):R774-R788. doi:10.1016/j.cub.2013.07.025

25. Wilckens KA, Woo SG, Kirk AR, Erickson KI, Wheeler ME. Role of sleep continuity and total sleep time in executive function across the adult lifespan. Annu Rev Psychol. 2013;64:135-168. doi:10.1146/ annurev-psych-113011-143750

26. Diamond A. Executive functions. Psychol Aging. 2014;29(3):658-665. doi:10.1037/a0037234
Neuropsychiatric Disease and Treatment

\section{Publish your work in this journal}

Neuropsychiatric Disease and Treatment is an international, peerreviewed journal of clinical therapeutics and pharmacology focusing on concise rapid reporting of clinical or pre-clinical studies on a range of neuropsychiatric and neurological disorders. This journal is indexed on PubMed Central, the 'PsycINFO' database and CAS,

\section{Dovepress}

and is the official journal of The International Neuropsychiatric Association (INA). The manuscript management system is completely online and includes a very quick and fair peer-review system, which is all easy to use. Visit http://www.dovepress.com/testimonials.php to read real quotes from published authors. 